\title{
A COMPARISON THEOREM FOR SELFADJOINT OPERATORS
}

\author{
AMIN BOUMENIR
}

(Communicated by Palle E. T. Jorgensen)

\begin{abstract}
In this work we shall establish a result concerning the spectral theory of differential operators. Let $L_{1}$ and $L_{2}$ be two self-adjoint operators acting in two different Hilbert spaces. Then under some conditions we shall prove that
\end{abstract}

$$
\left(d \Gamma_{1} / d \Gamma_{2}\right)\left(L_{2}\right)=\bar{V} V^{\prime},
$$

where $\Gamma_{1}(\lambda)$ and $\Gamma_{2}(\lambda)$ are the spectral functions associated with $L_{1}$ and $L_{2}$ respectively. $V$ is the shift operator mapping the set of generalized eigenfunctions of $L_{1}$ into the set of generalized eigenfunctions of $L_{2}$, that is

$$
y=V \varphi,
$$

where $L_{2} y=\lambda y$ and $L_{1} \varphi=\lambda \varphi$.

\section{INTRODUCTION}

As we shall be manipulating eigenfunctions we need to recall the theory of operators in rigged Hilbert spaces. Let $\Phi$ be a nuclear space, $N$-space, that is a countably normed space $\Phi=\bigcap_{n>1} \Phi_{n}$, such that, for any $p$, there exists $n>p$ so that the embedding $\Phi_{n} \hookrightarrow \Phi_{p}$ is a Hilbert-Schmidt operator, (see [7]). We recall that an $N$-space is a perfect space, and so each bounded set is relatively compact.

We now come to some interesting applications of the above idea. Suppose that an operator $L$ is symmetric in a Hilbert space $H$. Assume that there exists an $N$-space $\Phi$ (perfect) invariant under the operator $L$ and such that $H$ can be obtained as a completion of $\Phi$ under the inner product of $H$. We shall assume that the embedding $\Phi \hookrightarrow H$ is the identity. Since

$$
\Phi \stackrel{L}{\rightarrow} \Phi
$$

we have

$$
\Phi^{\prime} \stackrel{L^{*}}{\rightarrow} \Phi^{\prime}
$$

Then, using the symmetry of $L$, i.e., $L \subset L^{*}$, and the fact that

$$
\Phi \hookrightarrow H \hookrightarrow \Phi^{\prime},
$$

Received by the editors October 27, 1989.

1980 Mathematics Subject Classification (1985 Revision). Primary 46XXX.

(C) 1991 American Mathematical Society $0002-9939 / 91 \$ 1.00+\$ .25$ per page 
we obtain that $L^{*}=L$ on $\Phi$, and $L^{*}$ is seen as an extension of $L$ to $\Phi^{\prime}$. Thus we shall agree to denote by $L$ the operator $L^{*}$, and so the definition:

Definition 1. We shall say that a linear functional $\varphi \in \Phi^{\prime}$ is a generalized eigenfunction or eigenfunctional if

$$
L \varphi=\lambda \varphi \quad \text { in } \Phi^{\prime} .
$$

Below we recall a well known result, see [5, vol. 3 ].

Result. Let $L$ be a symmetric linear operator which is defined on $\Phi$ and maps $\Phi$ into itself. Assume that $\Phi$ is an $N$-space and that $L$ admits a self-adjoint extension to the Hilbert space $H$. Then $L$ possesses a complete system of eigenfunctionals in the space $\Phi^{\prime}$.

Let $L$ be a self-adjoint operator with simple spectrum acting in a separable Hilbert space $H$. If $\varphi(\lambda)$ are the eigenfunctionals, that is $L \varphi(\lambda)=\lambda \varphi(\lambda)$ in $\Phi^{\prime}$, then the associated isometry or $\varphi$-Fourier transform is given by

$$
\hat{f}(\lambda)=\langle f, \varphi(\lambda)\rangle_{\Phi \times \Phi^{\prime}} \quad \forall f \in \Phi
$$

and the inverse is

$$
f=\int \overline{\hat{f}(\lambda)} \varphi(\lambda) d \Gamma(\lambda) \in H .
$$

$\Gamma(\lambda)$ is a nondecreasing function and is called the spectral function. The Parseval equality reads

$$
\forall f, \forall \psi \in \Phi \quad(f(x), \psi(x))_{H}=\int \hat{f}(\lambda) \overline{\hat{\psi}(\lambda)} d \Gamma(\lambda) .
$$

Let us agree on some notations. Let $L_{1}$ and $L_{2}$ be two self-adjoint operators with simple spectrum and acting in two separable Hilbert spaces $H_{1}$ and $H_{2}$ respectively.

We assume the existence of two (perfect) $N$-spaces $\Phi_{1}$ and $\Phi_{2}$ such that

$$
\Phi_{i} \stackrel{L_{i}}{\rightarrow} \Phi_{i}, \quad i=1,2 .
$$

In all that follows, $\left\{\Phi_{i}, H_{i}, \Phi_{i}^{\prime}\right\}$ and $\Gamma_{i}(\lambda)$ will denote, respectively, the rigged spaces and the spectral functions associated with the self-adjoint operator $L_{i}$, where $i=1,2$. Denote by $\varphi(\lambda)$ and $y(\lambda)$ the generalized eigenfunctionals defined by

$$
\begin{aligned}
& L_{1} \varphi(\lambda)=\lambda \varphi(\lambda) \quad \text { in } \Phi_{1}^{\prime} \\
& L_{2} y(\lambda)=\lambda y(\lambda) \quad \text { in } \Phi_{2}^{\prime} .
\end{aligned}
$$

$\sigma_{i}$ denotes the spectrum of $L_{i}$, for $i=1,2$. The Fourier transform in this case is given by

$$
\begin{array}{ll}
f \in \Phi_{1} & \hat{f}^{1}(\lambda) \equiv\langle f, \varphi(\lambda)\rangle_{\Phi_{1} \times \Phi_{1}^{\prime}}, \\
\psi \in \Phi_{2} & \hat{\psi}^{2}(\lambda) \equiv\langle\psi, y(\lambda)\rangle_{\Phi_{2} \times \Phi_{2}^{\prime}} .
\end{array}
$$


In order to compare operators, we shall need to establish a correspondence between the two sets of eigenfunctionals. Assume the existence of a one-to-one mapping between the real sets $\sigma_{1}$ and $\sigma_{2}$, namely,

$$
T: \sigma_{2} \rightarrow \sigma_{1} \text {. }
$$

Definition 2. Let $\varphi(\lambda)$ and $y(\lambda)$ be the eigenfunctionals defined by (1.1), and let $T: \sigma_{2} \rightarrow \sigma_{1}$ be a one-to-one mapping. Then $V$ is said to be a shift operator if

$$
V \varphi(T(\lambda))=y(\lambda) \quad \forall \lambda \in \sigma_{2} .
$$

Remark. It is clear that the shift operator $V$ is a one-to-one mapping between the sets $\{\varphi(\lambda)\}_{\sigma_{1}}$ and $\{y(\lambda)\}_{\sigma_{2}}$. Hence it is defined on $\{\varphi(\lambda)\}_{\sigma_{1}}$, a subset of $\Phi_{1}^{\prime}$. We next extend $V$ to the algebraic span of $\{\varphi(\lambda)\}_{\sigma_{1}}$. For our immediate use, we shall only need the fact that $V$ is densely defined. Indeed, from the reflexivity of $\Phi_{1}$ and the completeness of $\{\varphi(\lambda)\}_{\sigma_{1}}$, the space spanned by $\{\varphi(\lambda)\}_{\sigma_{1}}$ is dense in $\Phi_{1}^{\prime}$. Therefore $V$ is densely defined. This enables us to define the adjoint operator $V^{\prime}$. By definition we have

$$
\langle\psi, V f\rangle_{\Phi_{2}^{\prime \prime} \times \Phi_{2}^{\prime}}=\left\langle V^{\prime} \psi, f\right\rangle_{\Phi_{1}^{\prime \prime} \times \Phi_{1}^{\prime}}
$$

Since the spaces are reflexive,

$$
\langle\psi, V f\rangle_{\Phi_{2} \times \Phi_{2}^{\prime}}=\left\langle V^{\prime} \psi, f\right\rangle_{\Phi_{1} \times \Phi_{1}^{\prime}}
$$

The domain of $V^{\prime}$ is defined by

$$
D_{V^{\prime}} \equiv\left\{\psi \in \Phi_{2} \mid f \rightarrow\langle\psi, V f\rangle \text { is continuous }\right\} .
$$

However there is a simple connection between $D_{V^{\prime}}$ and $\Phi_{2}$, indeed, we have the well-known result that, for example see [6, Chapter 2],

$$
V \text { admits closure } \Leftrightarrow D_{V^{\prime}} \text { is dense in } \Phi_{2} \text {. }
$$

\section{THE FACTORIZATION THEOREM}

Let us start with some notations. We know that $V^{\prime}$ acts between $\Phi_{2}$ and $\Phi_{1}$, which are imbedded in $H_{2}$ and $H_{1}$ respectively. Hence $V^{\prime}$ has a natural extension as an operator from $H_{2}$ into $H_{1}$, which we shall denote by $\widetilde{V}^{\prime}$. Thus

and, for $f \in D_{V^{\prime}}, \widetilde{V}^{\prime} f=V^{\prime} f$ in $H_{1}$.

$$
H_{2} \stackrel{\widetilde{V}^{\prime}}{\rightarrow} H_{1}
$$

Define an operator $G$ in $\Phi_{2}$ by

$$
G f=\int \overline{\hat{f}^{2}(\lambda)} y(\lambda) d \Gamma_{1}(T(\lambda)),
$$

where $\Gamma_{1}(\lambda)$ is the spectral function of $L_{1}$, and $T(\cdot)$ is the mapping used in the definition of the shift operator. The domain of $G$ is $D_{G}=\left\{f \in \Phi_{2} \mid G f \in \Phi_{2}^{\prime}\right\}$. Clearly if $\hat{f}^{2}(\lambda)$ has a compact support then $G f$ is defined. It is possible to represent $G$ if we knew the behaviour of $\Gamma_{1}(T(\lambda))$. 
Theorem 3. Let $L_{1}$ and $L_{2}$ be two self-adjoint operators with simple spectrum, acting in the rigged Hilbert spaces $\Phi_{1} \hookrightarrow H_{1} \hookrightarrow \Phi_{1}^{\prime}$ and $\Phi_{2} \hookrightarrow H_{2} \hookrightarrow \Phi_{2}^{\prime}$ respectively. If the shift operator $V$ admits closure, then

$$
G=\bar{V} V^{\prime},
$$

where $\bar{V}$ denotes the closure of $V$.

Proof. Let us give the diagram of the operator $V$

$$
\begin{array}{ccccc}
\Phi_{1} & \hookrightarrow & H_{1} & \hookrightarrow & \Phi_{1}^{\prime} \\
V^{\prime} \uparrow & & & & \downarrow V \\
\Phi_{2} & \hookrightarrow & H_{2} & \hookrightarrow & \Phi_{2}^{\prime},
\end{array}
$$

where $V \phi(T(\lambda))=y(\lambda)$.

Let $f$ and $\psi$ be two arbitrary elements of $D_{V^{\prime}}$.

$$
\begin{aligned}
\hat{f}^{2}(\lambda) & =\langle f, y(\lambda)\rangle_{\Phi_{2} \times \Phi_{2}^{\prime}} \\
& =\left\langle f, V \varphi(T(\lambda)\rangle_{\Phi_{2} \times \Phi_{2}^{\prime}}=\left\langle V^{\prime} f, \varphi(T(\lambda))\right\rangle_{\Phi_{1} \times \Phi_{1}^{\prime}}=\widehat{V^{\prime} f^{1}}(T(\lambda))\right.
\end{aligned}
$$

and, similarly,

$$
\hat{\psi}^{2}(\lambda)={\widehat{V^{\prime}}}^{1}(T(\lambda)) .
$$

Observing that the right-hand side of equations $(2.1),(2.2)$ are the $\varphi$-Fourier transform of $V^{\prime} f$ and $V^{\prime} \psi$, respectively, we obtain by using the Parseval equality,

$$
\begin{aligned}
& \left(V^{\prime} f, V^{\prime} \psi\right)_{H_{1}}=\int \widehat{V^{\prime} f^{1}} \widehat{V^{\prime} \psi^{1}(\lambda)} d \Gamma_{1}(\lambda) . \\
& \int \widehat{V^{\prime} f^{1}}(T(\lambda)) \widehat{V^{\prime} \psi^{1}(T(\lambda))} d \Gamma_{1}(T(\lambda))=\int \hat{f}^{2}(\lambda) \overline{\hat{\psi}^{2}(\lambda)} d \Gamma_{1}(T(\lambda)) .
\end{aligned}
$$

Clearly,

$$
\begin{aligned}
\langle f, G \psi\rangle_{\Phi_{2} \times \Phi_{2}^{\prime}} & =\left\langle f, \int \overline{\hat{\psi}^{2}(\lambda)} y(\lambda) d \Gamma_{1}(T(\lambda))\right\rangle_{\Phi_{2} \times \Phi_{2}^{\prime}} \\
& =\int \overline{\hat{\psi}^{2}(\lambda)} \hat{f}^{2}(\lambda) d \Gamma_{1}(T(\lambda)) .
\end{aligned}
$$

Hence $\left(V^{\prime} f, V^{\prime} \psi\right)_{H_{1}}=\langle f, G \psi\rangle_{\Phi_{2} \times \Phi_{2}^{\prime}}$ which implies that $D_{V^{\prime}} \subset D_{G}$. Since the imbedding $\Phi_{1} \hookrightarrow H_{1} \hookrightarrow \Phi_{1}^{\prime}$ is the identity,

$$
\left(V^{\prime} f, V^{\prime} \psi\right)_{H_{1}}=\left\langle V^{\prime} f, V^{\prime} \psi\right\rangle_{\Phi_{1} \times \Phi_{1}^{\prime}} .
$$

Therefore

$$
\langle f, G \psi\rangle_{\Phi_{2} \times \Phi_{2}^{\prime}}=\left\langle V^{\prime} f, V^{\prime} \psi\right\rangle_{\Phi_{1} \times \Phi_{1}^{\prime}},
$$

and, since $V$ admits closure, we have

$$
\left\langle V^{\prime} f, V^{\prime} \psi\right\rangle_{\Phi_{1} \times \Phi_{1}^{\prime}}=\left\langle f, \bar{V} V^{\prime} \psi\right\rangle_{\Phi_{2} \times \Phi_{2}^{\prime}}
$$


and so $\langle f, G \psi\rangle_{\Phi_{2} \times \Phi_{2}^{\prime}}=\left\langle f, \bar{V} V^{\prime} \psi\right\rangle_{\Phi_{2} \times \Phi_{2}^{\prime}}$. Therefore for $\psi \in D_{V^{\prime}}, G \psi=$ $\bar{V} V^{\prime} \psi$ in $\Phi_{2}^{\prime}$, and so $D_{G}=D_{\bar{V} V^{\prime}}$.

Remark. We had to use the fact that $V$ admitted closure. We shall see that we do not need such an assumption if we took $\widetilde{V}^{\prime}$ instead of $V^{\prime}$. For that define

$$
\begin{aligned}
& \widetilde{G}: H_{2} \rightarrow H_{2} \\
& \widetilde{G} f \equiv \int \overline{\hat{f}^{2}(\lambda)} y(\lambda) d \Gamma_{1}(T(\lambda)),
\end{aligned}
$$

$D_{\widetilde{G}}=\left\{f \in H_{2} \mid \widetilde{G} f \in H_{2}\right\}$. Here the Fourier transform is extended to $H_{2}$ by taking the closure of the Fourier transform to $H_{2}$. It is clear that $D_{\widetilde{G}}$ is dense in $H_{2}$. To see that, take the dense set of smooth compactly supported functions in $L_{\Gamma_{2}(\lambda)}^{2}$. Then, using the inverse $y$-Fourier transform, we shall obtain a dense set in $H_{2}$, which is also contained in $D_{\widetilde{G}}$. Hence $D_{\widetilde{G}}$ is dense in $H_{2}$, and so $\widetilde{G}$ is densely defined. From (2.3),

$$
\left(V^{\prime} f, V^{\prime} \psi\right)_{H_{1}}=\int \hat{f}^{2}(\lambda) \overline{\hat{\psi}^{2}(\lambda)} d \Gamma_{1}(T(\lambda)) .
$$

Since $f$ and $\psi$ are also in $H_{2},\left(V^{\prime} f, V^{\prime} \psi\right)_{H_{1}}=\left(\widetilde{V}^{\prime} f, \widetilde{V}^{\prime} \psi\right)_{H_{1}}$ and

$$
\int \hat{f}^{2}(\lambda) \overline{\hat{\psi}^{2}(\lambda)} d \Gamma_{1}(T(\lambda))=(f, \widetilde{G} \psi)_{H_{2}} .
$$

Therefore

$$
\left(\widetilde{V}^{\prime} f, \widetilde{V}^{\prime} \psi\right)_{H_{1}}=(f, \widetilde{G} \psi)_{H_{2}} .
$$

It is readily seen that $\tilde{V}^{\prime}$ is densely defined. Indeed, since

$$
\left\|\tilde{V}^{\prime} f\right\|^{2}=\int\left|\hat{f}^{2}(\lambda)\right|^{2} d \Gamma_{1}(T(\lambda))
$$

the argument used for $D_{\widetilde{G}}$ will go through. $\widetilde{V}^{\prime}$ densely defined means that the adjoint operator is well defined and, by (2.4), we deduce that

$$
\left(f,\left[\tilde{V}^{\prime}\right]^{\prime} \widetilde{V}^{\prime} \psi\right)_{H_{2}}=(f, \widetilde{G} \psi)_{H_{2}} .
$$

Therefore $\widetilde{G}=\left[\widetilde{V}^{\prime}\right]^{\prime} \widetilde{V}^{\prime}$, and we have just proved

Theorem 4. Let $L_{1}$ and $L_{2}$ be two self-adjoint operators with simple spectrum, acting in the rigged Hilbert spaces $\Phi_{1} \hookrightarrow H_{1} \hookrightarrow \Phi_{1}^{\prime}$ and $\Phi_{2} \hookrightarrow H_{2} \hookrightarrow \Phi_{2}^{\prime}$, respectively. Then

$$
\widetilde{G}=\left[\tilde{V}^{\prime}\right]^{\prime} \widetilde{V}^{\prime}
$$


There exists a particular case where it is possible to obtain a simple representation of the operator $G$. For that we need

Definition 5. $\Gamma_{1}(T(\lambda))$ is said to be absolutely continuous with respect to $\Gamma_{2}(\lambda)$ (denoted by $\mathrm{ABS}-d \Gamma_{2}(\lambda)$ ) if there exists a $d \Gamma_{2}(\lambda)$-summable function $g(\lambda)$ such that

$$
\Gamma_{1}(T(\lambda))=\int_{-\infty}^{\lambda} g(\lambda) d \Gamma_{2}(\lambda) .
$$

(Notation: $g(\lambda)=\left(d \Gamma_{1}(T) / d \Gamma_{2}\right)(\lambda)$.

Let $g\left(L_{2}\right)$ be the operator defined by

$$
\begin{array}{ccc}
\Phi_{2} & \stackrel{g\left(L_{2}\right)}{\rightarrow} & H_{2} \\
f(x) & \rightarrow & g\left(L_{2}\right) f(x) \\
\hat{2} \downarrow & & \hat{2}^{-1} \\
\hat{f}^{2}(\lambda) & \rightarrow & g(\lambda) \hat{f}^{2}(\lambda)
\end{array}
$$

or $g\left(L_{2}\right) f(x)=\int g(\lambda) \overline{\hat{f}^{2}(\lambda)} y(\lambda) d \Gamma_{2}(\lambda)$. Its domain is given by

$$
D_{g\left(L_{2}\right)}=\left\{f \in \Phi_{2} \mid g(\lambda) \hat{f}^{2}(\lambda) \in L_{\Gamma_{2}}^{2}\right\} .
$$

Therefore $G f=I_{\Phi_{2}^{\prime}} g\left(L_{2}\right) f$, for any $f \in D_{g\left(L_{2}\right)}$, and where $I_{\Phi_{2}^{\prime}}$ is the imbedding from $\mathrm{H}_{2} \hookrightarrow \Phi_{2}^{\prime}$, the identity. So we can write, for any $f \in D_{g\left(L_{2}\right)}$,

$$
G f=g\left(L_{2}\right) f \text { in } \Phi_{2}^{\prime} .
$$

In this way $G$ is an extension of $g\left(L_{2}\right)$ to $\Phi_{2}^{\prime}$. We shall agree to write $G \equiv$ $g\left(L_{2}\right)$ in $\Phi_{2}^{\prime}$. Thus

Corollary 6. Let $L_{1}$ and $L_{2}$ be two self-adjoint operators with simple spectrum, acting in the rigged Hilbert spaces $\Phi_{1} \hookrightarrow H_{1} \hookrightarrow \Phi_{1}^{\prime}$ and $\Phi_{2} \hookrightarrow H_{2} \hookrightarrow \Phi_{2}^{\prime}$, respectively. Assume that $V$ admits closure. If the function $\Gamma_{1}(T(\lambda))$ is $A B S$ $d \Gamma_{2}(\lambda)$, i.e., $d \Gamma_{1}(T(\lambda))=g(\lambda) d \Gamma_{2}(\lambda)$. Then, for any $f \in D_{g\left(L_{2}\right)}$,

$$
g\left(L_{2}\right) f=\bar{V} V^{\prime} f \text { in } \Phi_{2}^{\prime},
$$

where $\bar{V}$ denotes the closure of $V$.

If $\tilde{g}\left(L_{2}\right)$ denotes the extension of $g\left(L_{2}\right)$ to $H_{2} \rightarrow H_{2}$, then $\tilde{g}\left(L_{2}\right)=\widetilde{G}$ in $\mathrm{H}_{2}$, and so from Theorem 4,

Corollary 7. Let $L_{1}$ and $L_{2}$ be two self-adjoint operators with simple spectrum, acting in the rigged Hilbert spaces $\Phi_{1} \hookrightarrow H_{1} \hookrightarrow \Phi_{1}^{\prime}$ and $\Phi_{2} \hookrightarrow H_{2} \hookrightarrow \Phi_{2}^{\prime}$, respectively. If the function $\Gamma_{1}(T(\lambda))$ is $A B S-d \Gamma_{2}(\lambda)$, i.e., $d \Gamma_{1}(T(\lambda))=g(\lambda) d \Gamma_{2}(\lambda)$. Then

$$
\tilde{g}\left(L_{2}\right)=\left[\tilde{V}^{\prime}\right]^{\prime} \tilde{V}^{\prime} .
$$

We have defined $g\left(L_{2}\right)$ through the Fourier transform and claimed that it was the usual function of the operator $L_{2}$. Let us briefly show that the two 
definitions coincide. If $E_{\lambda}$ is the spectral family associated with $L_{2}$, then since the operator $L_{2}$ has a simple spectrum,

$$
d E_{\lambda} f=\overline{\hat{f}^{2}(\lambda)} y(x, \lambda) d \Gamma_{2}(\lambda) .
$$

Therefore

$$
g\left(L_{2}\right) f \equiv \int g(\lambda) d E_{\lambda} f=\int g(\lambda) \overline{\hat{f}^{2}(\lambda)} y(x, \lambda) d \Gamma_{2}(\lambda),
$$

and so the two definitions are in fact identical.

\section{General Results}

We have shown that $g\left(L_{2}\right)=\left[\tilde{V}^{\prime}\right]^{\prime} \tilde{V}^{\prime}$ on $D_{V^{\prime}}$. Clearly the boundedness of $g\left(L_{2}\right)$, which depends on the behaviour of $g(\lambda)$, must be related to the boundedness of $\widetilde{V}^{\prime}$.

Theorem 8. Assume that conditions in Corollary 7 hold; then $\widetilde{V}^{\prime}$ is a bounded operator $H_{2} \rightarrow H_{1}$ if and only if $\sqrt{g(\lambda)}$ is bounded $d \Gamma_{2}(\lambda)$ a.e.

Proof. Assume that $\sqrt{g(\lambda)}$ is bounded $d \Gamma_{2}(\lambda)$ a.e. Then there exists $M$ such that $|\sqrt{g(\lambda)}| \leq M d \Gamma_{2}(\lambda)$ a.e. From (2.4) we obtain

$$
\left\|\tilde{V}^{\prime} f\right\|_{H_{1}}=\left\|\sqrt{g(\lambda)} \hat{f}^{2}(\lambda)\right\|_{d \Gamma_{2}} \text { for } f \in \Phi_{2} \hookrightarrow H_{2},
$$

but $\left\|\sqrt{g(\lambda)} \hat{f}^{2}(\lambda)\right\|_{d \Gamma_{2}} \leq M\left\|\hat{f}^{2}(\lambda)\right\|_{d \Gamma_{2}} \leq M\|f\|_{H_{2}}$. Hence $\left\|\widetilde{V}^{\prime} f\right\|_{H_{1}} \leq M\|f\|_{H_{2}}$, which shows that $\widetilde{V}^{\prime}$ is a bounded operator from $H_{2}$ to $H_{1}$. Conversely, if $\widetilde{V}^{\prime}$ is bounded then, for any $f \in \Phi_{2}$, we do have

$$
\left\|\sqrt{g(\lambda)} \hat{f}^{2}(\lambda)\right\|_{d \Gamma_{2}}=\left\|\tilde{V}^{\prime} f\right\|_{H_{1}} \leq M\|f\|_{H_{2}} \leq M\left\|\hat{f}^{2}(\lambda)\right\|_{d \Gamma_{2}} .
$$

From the above inequality it is readily seen that $\sqrt{g(\lambda)}$ is $d \Gamma_{2}$ bounded.

Theorem 9. $\tilde{V}^{\prime}: H_{2} \rightarrow H_{1}$ is invertible if and only if

$$
\int_{k_{q}} d \Gamma_{2}(\lambda)=0
$$

where $k_{g} \equiv\{\lambda \mid g(\lambda)=0\}$ and

$$
\left\|\widetilde{V}^{\prime}\right\|=\underset{\lambda \in \sigma_{2}}{\operatorname{ess} \sup } \sqrt{g(\lambda)}
$$

From (2.4) we have that $\left\|\widetilde{V^{\prime}} f\right\|_{H_{1}}=\left\|\sqrt{g(\lambda)} \hat{f}^{2}(\lambda)\right\|_{d \Gamma_{2}}$. So the operator $\tilde{V}: H_{2} \rightarrow H_{1}$ is invertible if and only if $\sqrt{g\left(L_{2}\right)}$ is invertible. Thus we should have

$$
\sqrt{g\left(L_{2}\right)} f=0 \Rightarrow f=0 .
$$

Using the Fourier transform,

$$
\sqrt{g(\lambda)} \hat{f}^{2}(\lambda)=0 \Rightarrow \hat{f}^{2}(\lambda)=0 \quad d \Gamma_{2}(\lambda) \text { a.e. }
$$


As $\left\|\hat{f}^{2}\right\|$ depends on the support of $\Gamma_{2},(3.1)$ will have to be verified only on the support of $\Gamma_{2}$, or, in other words, on $\sigma_{2}$. Thus (3.1) means that there is no set of nonzero measure, where $g(\lambda)$ vanishes.

Suppose that we need to find $L_{1}$ from its spectral function $\Gamma_{1}$, i.e., the inverse spectral problem. Let $L_{2}$ be given with its spectral function $\Gamma_{2}$, and form the operator $G$. If we can solve $G=\bar{V} V^{\prime}$, then we claim that the inverse spectral problem is solved. Indeed, if we regard the eigenfunctions as a basis for the differential operator then the result is immediate,

$$
L_{2} y=\lambda y \quad \forall \lambda \in \sigma_{2} .
$$

So, by using the shift operator,

$$
\begin{gathered}
y=V \varphi \text { or } \varphi=V^{-1} y \\
L_{2} V \varphi=\lambda V \varphi
\end{gathered}
$$

and clearly

$$
V^{-1} L_{2} V \varphi=\lambda \varphi
$$

so that

$$
L_{1}=V^{-1} L_{2} V \text { in } \Phi_{1}^{\prime},
$$

which is exactly the formula for the change of basis. From (3.2) we can see that we can recover $L_{1}$ if $V^{-1}$ exists.

\section{THE SECOND FACTORIZATION}

Notice that the function $g(\lambda)$ in Definition 5 might not exist. In this section we shall give another way of relating the spectral functions. Let $T$ be a nondecreasing one-to-one one mapping between $\sigma_{1}$ and $\sigma_{2}$. As usual the shift operator is defined by $y(\lambda)=V \varphi(T(\lambda))$. Since $\Gamma_{1}(T(\lambda))$ and $\Gamma_{2}(\lambda)$ are nondecreasing functions we can assume the existence of an increasing function $s(\lambda)$ such that

$$
\Gamma_{1}(T(s(\lambda)))=\Gamma_{2}(\lambda) \text {. }
$$

With the help of $s(\lambda)$ we can define the following operator:

$$
\begin{array}{ccc}
\Phi_{2} & \stackrel{A_{s}}{\rightarrow} & H_{2} \\
f & \rightarrow & A_{s}(f) \\
\dot{i} \downarrow & & \uparrow^{-} 2^{-1} \\
\hat{f}^{2}(\lambda) & \rightarrow & \hat{f}^{2}(s(\lambda))
\end{array}
$$

The domain of $A_{s}$ is $D_{A_{s}}=\left\{f \in \Phi_{2} \mid \hat{f}^{2}(s(\lambda)) \in L_{\Gamma_{2}}^{2}\right\}$. Clearly $A_{s}={ }^{-} 2^{-1} s 0^{2}$, where so denotes the composition with the function $s(\lambda)$.

Denote by $\tilde{A}_{s}$ the closure of $A_{s}$ in $H_{2}$. 
Theorem 10. Let $L_{1}$ and $L_{2}$ be two self-adjoint operators having their spectral functions such that $\Gamma_{1}(T(s(\lambda)))=\Gamma_{2}(\lambda)$. Then

$$
\left[\tilde{V}^{\prime}\right]^{\prime} \widetilde{V}^{\prime}=\tilde{A}_{T s} \tilde{A}_{T s}
$$

Proof. Let $f$ and $\psi$ be two elements of $D_{\widetilde{V}^{\prime}}$. As usual we shall work with the Fourier transform. By (2.3),

$$
\begin{aligned}
\left(\widetilde{V}^{\prime} f, \widetilde{V}^{\prime} \psi\right)_{H_{1}} & =\int \hat{f}^{2}(\lambda) \overline{\hat{\psi}^{2}(\lambda)} d \Gamma_{1}(T(\lambda)) \\
& =\int \hat{f}^{2}\left(T(s(\lambda)) \overline{\hat{\psi}^{2}(T(s(\lambda)))} d \Gamma_{1}(T(s(\lambda)))\right. \\
& =\int \widehat{A_{T s}} f^{2}(\lambda) \widehat{A_{T s} \psi^{2}(\lambda)} d \Gamma_{2}(\lambda)=\left(\widetilde{A_{T s}} f, \widetilde{A_{T s}} \psi\right)_{H_{2}}
\end{aligned}
$$

From (4.2), we deduce that $D_{\widetilde{A}_{T s}}=D_{V^{\prime}}$ and $\widetilde{A}_{T s}$ is densely defined in $H_{2}$, so

$$
\left(f,\left[\widetilde{V}^{\prime}\right]^{\prime} \widetilde{V}^{\prime} \psi\right)_{H_{2}}=\left(f, \widetilde{A}_{T s} \widetilde{A}_{T s} \psi(x)\right)_{H_{2}} .
$$

Hence

$$
\left[\tilde{V}^{\prime}\right]^{\prime} \tilde{V}^{\prime}=\tilde{A}_{T s} \tilde{A}_{T s}
$$

Let us illustrate the next idea by an example. Let $s(\lambda)$ be an increasing function and define

$$
L_{1} \equiv s\left(L_{2}\right)
$$

It is clear that $L_{1} \varphi(\lambda)=\lambda \varphi(\lambda)$, where $\varphi(\lambda)=y\left(s^{-1}(\lambda)\right)$. Indeed

$$
s\left(L_{2}\right) y\left(s^{-1}(\lambda)\right)=s\left(s^{-1}(\lambda)\right) y\left(s^{-1}(\lambda)\right)=\lambda y\left(s^{-1}(\lambda)\right) .
$$

Therefore

$$
V y\left(s^{-1}(\lambda)\right)=y(\lambda)
$$

For any $f \in D_{V^{\prime}}$ we have

$$
\begin{aligned}
\langle f, y(\lambda)\rangle_{\Phi_{2} \times \Phi_{2}^{\prime}} & =\left\langle f, V y\left(s^{-1}(\lambda)\right)\right\rangle_{\Phi_{2} \times \Phi_{2}^{\prime}} \\
& =\left\langle V^{\prime} f, y\left(s^{-1}(\lambda)\right)\right\rangle_{\Phi_{1} \times \Phi_{1}^{\prime}}, \\
\hat{f}^{2}(\lambda) & ={\widehat{V^{\prime} f}}^{2}\left(s^{-1}(\lambda)\right),
\end{aligned}
$$

or

$$
\hat{f}^{2}(s(\lambda))={\widehat{V^{\prime}}}^{2}(\lambda),
$$

and so, taking the inverse $y$-Fourier transform, for any $f \in D_{V^{\prime}}, V^{\prime} f=\tilde{A}_{s} f$ holds in $\mathrm{H}_{2}$. In other words

$$
\widetilde{V}^{\prime} f=\tilde{A}_{s} f
$$


The spectral functions are

$$
\begin{aligned}
f & =\int \overline{\hat{f}^{1}(\lambda)} \varphi(\lambda) d \Gamma_{1}(\lambda)=\int \overline{\hat{f}^{2}\left(s^{-1}(\lambda)\right)} y\left(s^{-1}(\lambda)\right) d \Gamma_{1}(\lambda) \\
& =\int \overline{\hat{f}^{2}(\lambda)} y(\lambda) d \Gamma_{1}(s(\lambda)) .
\end{aligned}
$$

On the other hand,

$$
f=\int \overline{\hat{f}^{2}(\lambda)} y(\lambda) d \Gamma_{2}(\lambda)
$$

Therefore

$$
d \Gamma_{1}(s(\lambda))=d \Gamma_{2}(\lambda) .
$$

Theorem 11. Let $L_{2}$ be a self-adjoint operator acting in the rigged Hilbert space $\Phi_{2} \hookrightarrow H_{2} \hookrightarrow \Phi_{2}^{\prime}$. If $L_{1}=s\left(L_{2}\right)$, where $s$ is an increasing function, then

$$
\Gamma_{1}(s(\lambda))=\Gamma_{2}(\lambda) \text { for any } f \in D_{\widetilde{V}}, \quad \widetilde{V}^{\prime} f=\tilde{A}_{s} f .
$$

We now discuss the case where we are given two spectral functions, $\Gamma_{1}(\lambda)$ and $\Gamma_{2}(\lambda)$ such that $\Gamma_{1}(s(\lambda))=\Gamma_{2}(\lambda)$, where $s(\lambda)$ is a one-to-one mapping $\sigma_{2} \rightarrow \sigma_{1}$. The operators are not supposed to commute, and so they are not functions of each other.

$\Gamma_{1}(s(\lambda))$ is ABS- $d \Gamma_{2}(\lambda)$ since $g(\lambda)=\left(d \Gamma_{1}(s) / d \Gamma_{2}\right)(\lambda)=1$, and so $\tilde{g}\left(L_{2}\right)=$ Id on $D_{g\left(L_{2}\right)} \subset H_{2} \rightarrow H_{2}$. The shift operator is given by

$$
V \varphi(s(\lambda))=y(\lambda) \quad \text { for } \lambda \in \sigma_{2} .
$$

From Corollary 4 we deduce that $\tilde{g}\left(L_{2}\right)=\left[\tilde{V}^{\prime}\right]^{\prime} \widetilde{V}^{\prime}$ or that $\mathrm{Id}=\left[\widetilde{V}^{\prime}\right]^{\prime} \tilde{V}^{\prime}$. Hence $\widetilde{V}^{\prime}$ is a unitary operator, in fact

$$
\left(\widetilde{V}^{\prime} f, \widetilde{V}^{\prime} \psi\right)_{H_{1}}=(f, \psi)_{H_{2}}
$$

We can decompose $V$ into two shifts:

$$
\varphi(s(\lambda)) \stackrel{R}{\rightarrow} \varphi(\lambda) \stackrel{W}{\rightarrow} y(\lambda),
$$

so $V=W \cdot R$. By definition $R \varphi(s(\lambda))=\varphi(\lambda)$ or $R \varphi(\lambda)=\varphi\left(s^{-1}(\lambda)\right)$. So, by Theorem 11 , if $\widetilde{R}^{\prime}$ is the closure of $R^{\prime}$ in $H_{1}, \hat{f}^{1}\left(s^{-1}(\lambda)\right)={\widehat{R^{\prime} f}}^{1}(\lambda) \Rightarrow \widetilde{A}_{s-1}=$ $\widetilde{R}^{\prime}$. Therefore

$$
\mathrm{Id}=\left[\widetilde{V}^{\prime}\right]^{\prime} \widetilde{V}^{\prime}=\widetilde{W}^{\prime \prime} \widetilde{R}^{\prime \prime} \widetilde{R}^{\prime} \widetilde{W}^{\prime}=\left[\widetilde{W}^{\prime}\right]^{\prime}\left(\widetilde{A}_{s}-1\right)^{\prime}\left(\tilde{A}_{s}-1\right) \widetilde{W}^{\prime} .
$$

\section{EXAMPLES}

Example. As an example we shall show how to apply the above ideas and obtain an extension of the Gelfand Levitan theory to the generalized second order differential operator $L_{2} \equiv-d^{2} / w(x) d x^{2}$, where $w(x) \geq 0$. Suppose we are given two second order differential operators such that Theorem 4 holds:

$$
L_{1}=L_{2}+q(x)
$$


where

$$
L_{2}=-\frac{d^{2}}{w(x) d x^{2}},
$$

$w(x) \geq 0$, and the boundary conditions are included in the definition of the operators. Notice that the operators $L_{1}$ and $L_{2}$ act in the same space $L_{w(x) d x}^{2}(0, \infty)$. In what concerns the rigged Hilbert space structure we refer to the construction done by Aleksandrjian. Or, since the Fourier transform is defined, one can simply take $\Phi \equiv \hat{S}^{-1}$ where $S$ is a space of rapidly decreasing functions which is nuclear and invariant by the multiplication by $\lambda$. In this way $\Phi$ is also an $N$-space. The shift operator is given by

$$
V=1+H
$$

where

$$
1(f)=f
$$

and

$$
H(f) \equiv \int_{0}^{x} H(x, t) f(t) w(t) d t .
$$

The relation between the functions $H(x, t)$ and $q(x)$ is as follows: we have shown in (3.2) that

$$
L_{2} V=V L_{1} \quad \text { in } \Phi_{2}^{\prime}
$$

or

$$
L_{2}(1+H)=(1+H)\left(L_{2}+q\right) .
$$

So $L_{2} H-H L_{2}=q+H q$, which is a hyperbolic equation, and $G=V V^{\prime}$ or, in other words,

$$
G=1+H+H^{\prime}+H H^{\prime}
$$

which means that

$$
\{G-1\} f(x)=\left\{H+H^{\prime}+H H^{\prime}\right\} f(x)
$$

where $f(x)$ is a smooth function with compact support. Now notice that the term on the left-hand side of (5.2) is nothing other than

$$
[G-1] f(x)=\int \hat{f}^{2}(\lambda) y(x, \lambda) d\left[\Gamma_{1}(\lambda)-\Gamma_{2}(\lambda)\right]
$$

Now, using the expression of the Fourier transform, we have

$$
\hat{f}^{2}(\lambda)=\int f(t) y(t, \lambda) w(t) d t .
$$

Assuming that

$$
P(x, t) \equiv \int y(t, \lambda) y(x, \lambda) d\left[\Gamma_{1}-\Gamma_{2}\right](\lambda)
$$


is a continuous function of $t$ and $x$ and, using the fact that $f(x)$ is of compact support, we obtain, by applying Fubini's theorem,

$$
\begin{aligned}
{[G-1] f(x) } & =\int f(t)\left[\int y(t, \lambda) y(x, \lambda) d\left[\Gamma_{1}-\Gamma_{2}\right](\lambda)\right] w(t) d t \\
& =\int f(t) P(t, x) w(t) d t .
\end{aligned}
$$

We can now express the right-hand side of (5.2):

$$
\begin{aligned}
{\left[H+H^{\prime}+H H^{\prime}\right] f(x)=} & \int_{0}^{x} H(x, t) f(t) w(t) d t+\int_{x}^{\infty} H(t, x) f(t) w(t) d t \\
& +\int_{0}^{x} H(x, s) \int_{s}^{\infty} H(s, t) f(t) w(t) d t w(s) d s .
\end{aligned}
$$

The last term can be written as

$$
\begin{aligned}
\int_{0}^{x} & \int_{0}^{x} H(x, s) H(s, t) w(s) d s f(t) w(t) d t \\
& +\int_{x}^{\infty} \int_{0}^{x} H(x, s) H(s, t) w(s) d s f(t) w(t) d t,
\end{aligned}
$$

and so in the weak sense we do have the result

$$
P(t, x)=H(x, t)+\int_{0}^{x} H(x, s) H(s, t) w(t) d t, \quad t<x .
$$

Suppose that $V^{-1}$ exists and $V^{-1} \equiv 1+K$. Then we do have

$$
\begin{aligned}
V^{-1} G & =V^{\prime} \\
(1+K) G & =1+H^{*} \\
(1+K) G f(x) & =\left(1+H^{*}\right) f(x)
\end{aligned}
$$

$$
G f(x)+\int_{0}^{x} K(x, t) G f(t) w(t) d t=f(x)+\int_{x}^{\infty} H(t, x) f(t) w(t) d t
$$

or

$$
\begin{aligned}
& {[G-1] f(x)+\int_{0}^{x} K(x, t) f(t) w(t) d t+\int_{0}^{x} K(x, t)[G-1] f(t) w(t) d t} \\
& \quad=\int_{x}^{\infty} H(t, x) f(t) w(t) d t .
\end{aligned}
$$

But $[G-1] f(x)=\int P(t, x) f(t) w(t) d t$, so we have

$$
\begin{aligned}
& \int P(t, x) f(t) w(t) d t+\int_{0}^{x} K(x, t) f(t) w(t) d t \\
& \quad+\iint_{0}^{x} K(x, s) P(s, t) w(s) d s f(t) w(t) d t=\int_{x}^{\infty} H(t, x) f(t) w(t) d t
\end{aligned}
$$

Hence,

$$
P(t, x)+K(x, t)+\int_{0}^{x} K(x, s) P(s, t) w(s) d s=H(x, t) .
$$


Observe that $H(t, x)=0$ if $t<x$; hence

$$
P(t, x)+K(x, t)+\int_{0}^{x} K(x, s) P(s, t) w(s) d s=0
$$

where $0 \leq t<x$.

Example 2. Everitt and Zettl computed the Weyl-Titchmarsh function, associated with the operator

$$
L_{2} f \equiv \frac{-1}{x^{\alpha}} f^{\prime \prime}(x), \quad x \in[0, \infty)
$$

They proved that the spectral function associated with

$$
\begin{aligned}
& L_{2} f \equiv \frac{-1}{x^{\alpha}} \frac{d^{2}}{d x^{2}} f(x), \quad x \geq 0, \\
& f^{\prime}(0)=0
\end{aligned}
$$

is of the form

$$
\Gamma_{2}(\lambda)= \begin{cases}c \cdot \lambda^{(\alpha+1) /(\alpha+2)} & \text { for } \lambda \geq 0 \\ 0 & \text { for } \lambda<0 .\end{cases}
$$

For further details see [2].

Let us prove the same result but using our method. We shall define another operator $L_{1}$ and then obtain the shift operator $V$. Having $V$ we shall use $\left(d \Gamma_{1} / d \Gamma_{2}\right)\left(L_{2}\right)=V V^{\prime}$ to obtain an equation for $\Gamma_{1}(\lambda)$.

Denote by $y(x, \lambda)$ the eigenfunctions of $L_{2} y(x, \lambda)=\lambda y(x, \lambda)$

$$
\begin{aligned}
& y^{\prime \prime}(x, \lambda)+\lambda x^{\alpha} y(x, \lambda)=0 \\
& y(x, \lambda)=1 \text { and } y^{\prime}(x, \lambda)=0 .
\end{aligned}
$$

Solutions of $(5.11)$ can be written in terms of the Bessel functions $\left(\lambda=\mu^{2}\right)$ :

$$
y\left(x, \mu^{2}\right)=A r(x) \sqrt{t(x) \mu} J_{\nu}(t(x) \mu)+B r(x) \sqrt{t(x) \mu} J_{-\nu}(t(x) \mu),
$$

where $r(x)=x^{-\alpha / 4}, t(x)=2 \nu x^{1 / 2 \nu}$ and $\nu=1 /(\alpha+2) . \quad A$ and $B$ are determined from the boundary conditions

$$
y(0, \lambda)=1 \text { and } y^{\prime}(0, \lambda)=0 .
$$

So

$$
A=0 \text { and } B=\mu^{\nu-1 / 2} \cdot C(\nu),
$$

where $C(\nu)$ is a function of $\nu$ only.

Define an operator

$$
\begin{aligned}
L^{2} & \stackrel{T}{\rightarrow} L_{x}^{2} \alpha_{d x} \\
f & \rightarrow T f(x)=r(x) f(t(x)) .
\end{aligned}
$$

We do have $T T^{\prime}=$ Id. Since $T^{\prime} g(t)=x(t)^{\alpha / 4} g(x(t))$, where $x(t)$ is the inverse function of $t(x)$. Thus $y\left(x, \mu^{2}\right)=\mu^{\nu-1 / 2} \cdot C(\nu) \cdot T\left(\sqrt{t \mu} J_{-\nu}(t \mu)\right)$. 
Now it is time to find the operator $L_{1}$. If

$$
\varphi\left(t, \mu^{2}\right)=\sqrt{t \mu} J_{-\nu}(t \mu)
$$

are the eigenfunctionals of $L_{1}$, then, from the inversion formula of the Bessel functions,

$$
\begin{aligned}
F(\lambda) & =\int_{0}^{\infty} f(t) \sqrt{t \sqrt{\lambda}} J_{-\nu}(t \sqrt{\lambda}) d t \\
f(t) & =\int_{0}^{\infty} F(\lambda) \sqrt{t \sqrt{\lambda}} J_{-\nu}(t \sqrt{\lambda}) d \sqrt{\lambda}
\end{aligned}
$$

We deduce that $d \Gamma_{1}(\lambda)=d \sqrt{\lambda}$.

The shift operator is given by

$$
y\left(x, \mu^{2}\right)=\mu^{\nu-1 / 2} \cdot C(\nu) \cdot T\left[\varphi\left(x, \mu^{2}\right)\right],
$$

since

$$
y(x, \lambda)=\lambda^{(\nu-1 / 2) / 2} \cdot C(\nu) \cdot T[\varphi(x, \lambda)] .
$$

Using the fact that $L_{2} y=\lambda y$ we have

$$
\begin{aligned}
L_{2}^{(\nu-1 / 2) / 2} y & =\lambda^{(\nu-1 / 2) / 2} y \\
& =\lambda^{(\nu-1 / 2) / 2} \cdot C(\nu) \cdot L_{2}^{(\nu-1 / 2) / 2}=T[\varphi]
\end{aligned}
$$

Simplifying by $\lambda^{(\nu-1 / 2) / 2}$,

$$
y(x, \lambda)=C(\nu) \cdot L_{2}^{(\nu-1 / 2) / 2} \cdot T[\varphi] .
$$

Recall that

$$
y \equiv V \varphi,
$$

hence

$$
V[\cdot]=C(\nu) \cdot L_{2}^{(\nu-1 / 2) / 2} \cdot T[\cdot] .
$$

From Theorem 4 we obtain that

$$
\frac{d \Gamma_{1}}{d \Gamma_{2}}\left(L_{2}\right)=V V^{\prime}=C(\nu)^{2} \cdot L_{2}^{(\nu-1 / 2) / 2} \cdot T \cdot T^{\prime} L_{2}^{(\nu-1 / 2) / 2} .
$$

Since $T T^{\prime}=1$

$$
\frac{d \Gamma_{1}}{d \Gamma_{2}}\left(L_{2}\right)=C(\nu)^{2} \cdot L_{2}^{(\nu-1 / 2)}
$$

Hence

$$
\frac{d \Gamma_{1}}{d \Gamma_{2}}(\lambda)=C(\nu)^{2} \cdot \lambda^{(\nu-1 / 2)}
$$


Let us solve the above differential equation:

$$
\begin{aligned}
d \Gamma_{2}(\lambda) & =\frac{1}{C(\nu)^{2}} \lambda^{1 / 2-\nu} d \Gamma_{1}(\lambda) \\
& =\frac{1}{C(\nu)^{2}} \lambda^{1 / 2-\nu} d \sqrt{\lambda} \\
\Gamma_{2}(\lambda) & =\frac{1}{C(\nu)^{2}} \int_{0}^{\sqrt{\lambda}} s^{2(1 / 2-\nu)} d s \\
& =\frac{1}{2-2 \nu} \cdot \frac{1}{C(\nu)^{2}} \int_{0}^{\sqrt{\lambda}} d s^{2-2 \nu} \\
\Gamma_{2}(\lambda) & =\frac{1}{2-2 \nu} \cdot \frac{1}{C(\nu)^{2}} \lambda^{1-\nu} .
\end{aligned}
$$

So

$$
\Gamma_{2}(\lambda)=c \lambda^{1-1 /(\alpha+2)},
$$

where $c$ is a constant. That is what Everitt and Zettl have shown.

\section{REFERENCES}

1. Aleksandrjian, Spectral decomposition of arbitrary self-adjoint operators into eigenfunctionals, Soviet Mat. 5 (1985), 607-611.

2. W. N. Everitt and A. Zettl, A class of integral inequalities, J. London Math. Soc (2) 17 (1978), 291-303.

3. I. M. Gelfand and A. G. Kostyuchenko, Eigenfunction expansions of differential and other operators, Dokl. Akad. Nauk SSSR 103 (1955), 349-352.

4. I. M. Gelfand and B. M. Levitan, On the determination of a differential equation from its spectral function, Amer. Math. Soc. Transl. (2) 1 (1951), 239-253.

5. I.M. Gelfand and G. E. Shilov, Generalized functions, vols. 2-4, Academic Press, New York, 1961. (English transl.)

6. S. Goldberg, Unbounded operators; theory and applications, McGraw Hill, New York, 1966.

7. K. Maurin, General eigenfunctions expansions, Polska. Akad. Nauk. 48 (1969).

9. N. Naimark, Linear differential operators, Part 2, Ungar, New York, 1968. (English transl.)

10. A. I. Plesner and V. A. Rohlin, Spectral theory of linear operators, Amer. Math. Soc. Transl. (2) 62 (1946), 24-101.

11. J. Weidman, Spectral theory of differential operators, Lecture Notes in Math., vol. 1258, Springer-Verlag.

Department of Mathematics, King Fahd University, Dhahran, Saudi Arabia 